\title{
Effects of along- and cross-radar-beam winds on Doppler radar spectrum
}

\author{
Y.-H. Chu ${ }^{1}$ \\ ${ }^{1}$ Institute of Space Science/Center for Space and Remote Sensing Research, National Central University, Chung-Li, Taiwan
}

Received: 27 August 2004 - Revised: 30 August 2004 - Accepted: 21 December 2004 - Published: 30 March 2005

\begin{abstract}
Beam broadening effects on the Doppler radar spectrum induced by along- and cross-radar-beam winds are theoretically investigated in this article. Analytical expression of beam broadening spectrum for a vertical beam subject to a constant vertical wind in the absence of horizontal wind is derived first. We find that the resultant beam broadening spectral shape is in an exponential form, rather than a Gaussian shape. The peak of the exponential spectrum corresponds to the true vertical wind velocity, and the spectral width $\sigma$ is a function of vertical wind velocity $w$ and the halfpower beam width $\theta_{1 / 2}$ of the radar beam, in accordance with the expression $\sigma=0.09 \mathrm{w} \theta_{1 / 2}^{2}$. The beam broadening spectrum for a vertical radar beam subject to both vertical and horizontal winds is numerically studied, and the result shows that the spectral shape is distorted from the Gaussian form. The estimated vertical wind velocity of the distorted spectrum is very close to the true vertical wind velocity with a difference of less than $0.6 \%$ for a typical case. However, its spectral width is substantially greater than that of the conventional Gaussian spectrum broadened by horizontal wind only. The difference of the spectral widths $\Delta \sigma$ (in unit of percentage) in the two, as a function of the half-power beam width and the ratio of vertical to horizontal wind velocities, can be well approximated by a parabolic equation. With this equation and the conventional Gaussian beam broadening spectral width caused by horizontal wind, the true width of the distorted beam broadening spectrum induced by vertical and horizontal winds can be inferred without complicated numerical computation. Through a transformation of wind velocity from vertical and horizontal winds into alongand cross-radar-beam winds defined on the cross section of an oblique radar beam, the results obtained from the case of vertical radar beam can be directly applied to the case of the oblique beam. We show that in some special cases the beam broadening spectral widths of an oblique beam subject to both vertical and horizontal winds will be exceedingly narrower than those estimated by the existing formula, irrespective of the presence of strong horizontal wind velocity.
\end{abstract}

Correspondence to: Y.-H. Chu

(yhchu@jupiter.ss.ncu.edu.tw)
Keywords. Radio science (Atmospheric propagation) - Meteorology and atmospheric dynamics (Instruments and technique) - General or miscellaneous (technique applicable in three or more fields)

\section{Introduction}

It is well recognized that the background wind in the illuminating region of a Doppler radar with finite antenna beam width plays a crucial role in dominating the behavior of the Doppler spectrum for the radar returns generated from diffusive targets in the atmosphere, including turbulence-induced refractivity fluctuations and hydrometeors. The effect of the background wind on the Doppler radar spectrum is attributed to the fact that the diffusive targets drifting with background wind may have radial velocities depending on wind velocity and the angular positions of the targets in the scattering volume. As a result, the observed Doppler spectrum will be broadened owing to the radar returns consisting of the signals at various Doppler frequencies that correspond to the radial velocities of the targets located in the different positions in the scattering volume. This broadening effect on the breadth of the Doppler spectrum caused simply by background wind and the antenna beam width is called the beam broadening effect and the resulting Doppler spectrum, without containing the information of atmospheric targets, is termed as the beam broadening spectrum (Hocking, 1983). In this sense, the beam broadening spectrum can be interpreted as the result of the radar returns from idealized targets that are equal in size and isotropic in shape and distribute uniformly without random motion in the scattering volume. Obviously, the width of the beam broadening spectrum is a function of the antenna beam width and background wind velocity, and bears no relation to the characteristics of real atmospheric targets.

The relation between the beam broadening spectrum and the observed Doppler spectra from various atmospheric targets have long been the topics of interest in the Doppler radar community. For example, considering the beam broadening effect, Hocking (1983) established a method to estimate clear-air turbulent strength from the observed Doppler 
spectral width. Wakasugi et al. (1986) proposed a deconvolution algorithm to remove clear-air turbulence and beam broadening effects from the observed precipitation Doppler spectrum to correctly estimate the raindrop size distribution. With the help of the beam broadening effect on clear-air turbulent spectrum, Chu et al. (1990) developed a method to measure the aspect sensitivity of VHF backscatter made by a vertically pointed radar beam with a relatively large beam width. Recently, Nastrom and Eaton (1997) proposed a correction formula, in which the effects of beam broadening, gravity wave broadening and wind shear broadening are considered, to estimate the turbulence eddy dissipation coefficient from the oblique Doppler spectral width to avoid the plausible spectral narrowing effects due to specular reflections.

Although the beam broadening effects on the Doppler spectra for various targets have been investigated extensively for years, almost all of the studies take advantage of beam broadening spectrum due to horizontal wind and ignore the plausible contributions of vertical wind (or along-radar-beam wind) to the Doppler spectral width (Sloss and Atlas, 1968; Hocking, 1986; Nastrom, 1997). To the author's knowledge, the investigation of along-radar-beam wind effects on the shape and width of the Doppler radar spectrum has not been documented in literature. For the vertical radar beam, the effect of the along-radar-beam (or vertical) wind on the Doppler spectral width can be ignored for quiet conditions. This is because under this condition the vertical air velocity is very weak (less than a few $\mathrm{m} \mathrm{s}^{-1}$ ) and the ratio of vertical $w$ to horizontal $U$ wind velocity is very small (in order of magnitude of $10^{-2}$ ), leading to the corresponding beam broadening spectral width which is 2 orders of magnitude smaller than that caused by horizontal wind (Chu, 2002). However, it may not be the case for an oblique radar beam under disturbed conditions. Observations made by radars and aircrafts both show that the magnitude of the vertical wind velocity associated with a thunderstorm can be as large as $20 \mathrm{~m} \mathrm{~s}^{-1}$ (Batton and Theiss, 1966; Brandes et al., 1995). Moreover, considerably strong vertical wind velocity associated with a thunderstorm usually is accompanied by a weak horizontal flow to favor the growth of the storm, suggesting a very large ratio of $w$ to $U$ (Nelson, 1983; Ziegler, 1983; Brandes et al., 1995). Therefore, in the presence of intense vertical wind velocity and/or a large ratio of $w$ to $U$, one might expect that the effects of vertical wind on the Doppler radar spectrum for a oblique radar beam may be substantial and should be taken into account in interpreting the behavior of the observed Doppler spectral width. One of the objectives of this research is to quantitatively investigate to what extent is the effect of the vertical wind.

Generally, the contour of radial velocity in resolution volume is treated as a straight line to facilitate the mathematical manipulation in the theoretical derivation of the beam broadening spectral width (Sloss and Atlas, 1968; Nastrom, 1997; Chu, 2002). Analysis indicates that this straight line approximation is valid under the conditions of a low ratio of $w$ to $U$ (smaller than about 0.3 ), together with a slight tilt angle of the oblique beam (less than about $\left.20^{\circ}\right)(\mathrm{Chu}, 2002)$. However, for the conditions of a high ratio of $w$ to $U$ and a large zenith angle of the radar beam, the curved nature of the radial velocity contour should be taken into account in the theoretical development of the corresponding beam broadening spectral width, and the shape of the beam broadening spectrum will be non-Gaussian. Numerical results presented in this article show that the estimated width of the nonGaussian beam broadening spectrum is greater than that calculated from the conventional Gaussian spectrum, implying that the atmospheric parameters retrieved from the observed Doppler spectral width will be overestimated if the conventional Gaussian beam broadening spectrum is employed for the estimation.

This article is an attempt to theoretically study the effects of along-radar-beam and cross-radar-beam winds on the behavior of the Doppler radar spectrum for vertical and oblique radar beams. We find that the shape of the beam broadening spectrum for a vertical radar beam subject to a vertical wind is in an exponential form, with a tail extending toward the lower Doppler frequency end, and its spectral width (defined as the second moment of the spectrum) as a function of the product of the vertical wind velocity and the square of the half-power beam width which is considerably smaller than that caused by the horizontal wind. Moreover, we also show in this article that the beam broadening spectral width of an oblique beam may be exceedingly narrow, if the radar beam is subject to specific vertical and horizontal winds such that the composite cross-radar-beam wind velocity is negligibly small and the along-radar-beam wind velocity is relatively large.

This paper is organized as follows. The analytical expression of the beam broadening spectrum caused by a vertical wind in the absence of the horizontal wind will be derived theoretically in Sect. 2. In Sect. 3, the beam broadening spectrum of a vertical beam induced by a horizontal wind, in combination with vertical winds, is investigated. In light of the difficulty in the theoretical derivation of the exact expression, a numerical simulation technique is developed in this section to obtain the desired beam broadening spectrum for different combinations of vertical and horizontal winds. Empirical relations between estimation errors of the vertical wind velocity and the beam broadening spectral width are established in this section as well. The transformation of wind velocity for the application of the vertical beam results to the case of the oblique beam is made in Sect. 4. Section 5 contains the discussion and Sect. 6 is the conclusion.

\section{Beam broadening spectrum caused by vertical wind}

In this section, an analytical expression of the beam broadening spectrum for a vertical radar beam subject to vertical wind is derived. In light of the fact that the beam broadening spectrum is governed only by background wind and radar beam width, the characteristics of the atmospheric target are not considered in the derivation. Moreover, the background 
wind velocity is assumed to be constant and uniform in the resolution volume and no random fluctuation of the velocity is included. In addition, the scatterers with equal size and isotropic shape are assumed to distribute uniformly in resolution volume, leading to a uniform reflectivity across the radar beam. Although a vertically pointed radar beam is considered here, we will show in Sect. 4 that the results obtained in this section can directly apply to the case of the oblique beam through appropriate transformation of wind velocities.

It is noteworthy that the analytical expression of the beam broadening spectrum can theoretically be obtained by integrating radar power along the contour of the constant radial velocity (or Doppler frequency) in the resolution volume. Mathematically, the total power received by a vertically pointed radar in a given Doppler frequency shift interval from $f$ to $f+d f$ can be formulated below (Sloss and Atlas, 1968; Chu, 2002)

$S_{V}(f) d f=\int \Pi_{0} g_{0} G_{b}(\theta, \Phi) d A(\theta, \Phi)$,

where $S_{V}(f)$ is the beam broadening power spectral density, $\Pi_{0}$ is reflectivity that is assumed to be uniformly distributed in the resolution volume, $g_{0}=C / R^{2}$ is for the far field condition and $C$ is a constant determined by radar parameters, including transmitted power, radar wavelength, radar efficiency, and so on, $G_{b}(\theta, \Phi)$ is two-way antenna beam power pattern, $d A$ is an infinitesimal area at coordinate $(\theta, \Phi), f$ is the Doppler frequency shift, $\theta$ is zenith angle, and $\Phi$ is the azimuth angle with respect to the reference axis. The key issue in deriving the beam broadening spectrum is to find the mathematical relations between $\theta, \Phi$ and $f$. Figure 1 presents the geometric relationship between vertically pointed antenna beam and the vertical wind $w$, in which the horizontal plane defined by axes $X$ (toward due east) and $Y$ (toward due north) represents a cross section of radar volume and axis $X$ is adopted as a reference axis in the following derivation. Let point $\boldsymbol{B}$ be located on the plane with angular coordinate $(\theta, \Phi)$. From Fig. 1, the relations between $(x, y)$ and $(\theta, \Phi)$ can be expressed as follows:

$x=R \tan \theta \cos \Phi$,

$y=R \tan \theta \sin \Phi$,

where $R$ is the height of the horizontal plane. It is easy to show that the area dA of an infinitesimal region for a quadrilateral $\mathrm{BCDE}$ can be represented in terms of $R, \theta$ and $\Phi$ as follows:

$d A=R^{2} \tan \theta d \theta d \Phi$.

It is noteworthy from Fig. 1 that $d A$ defined in Eq. (4) represents an infinitesimal area of a circular annulus in the radar beam in the angular range $\theta-\theta+d \theta$. To proceed, a Gaussian antenna beam pattern with circular symmetry for a monostatic radar is assumed and its two-way antenna beam power pattern in the rectangular coordinate is given below to simplify the mathematical manipulation

$G_{b}(x, y)=e^{-\left(x^{2}+y^{2}\right) / \sigma^{2}}$.

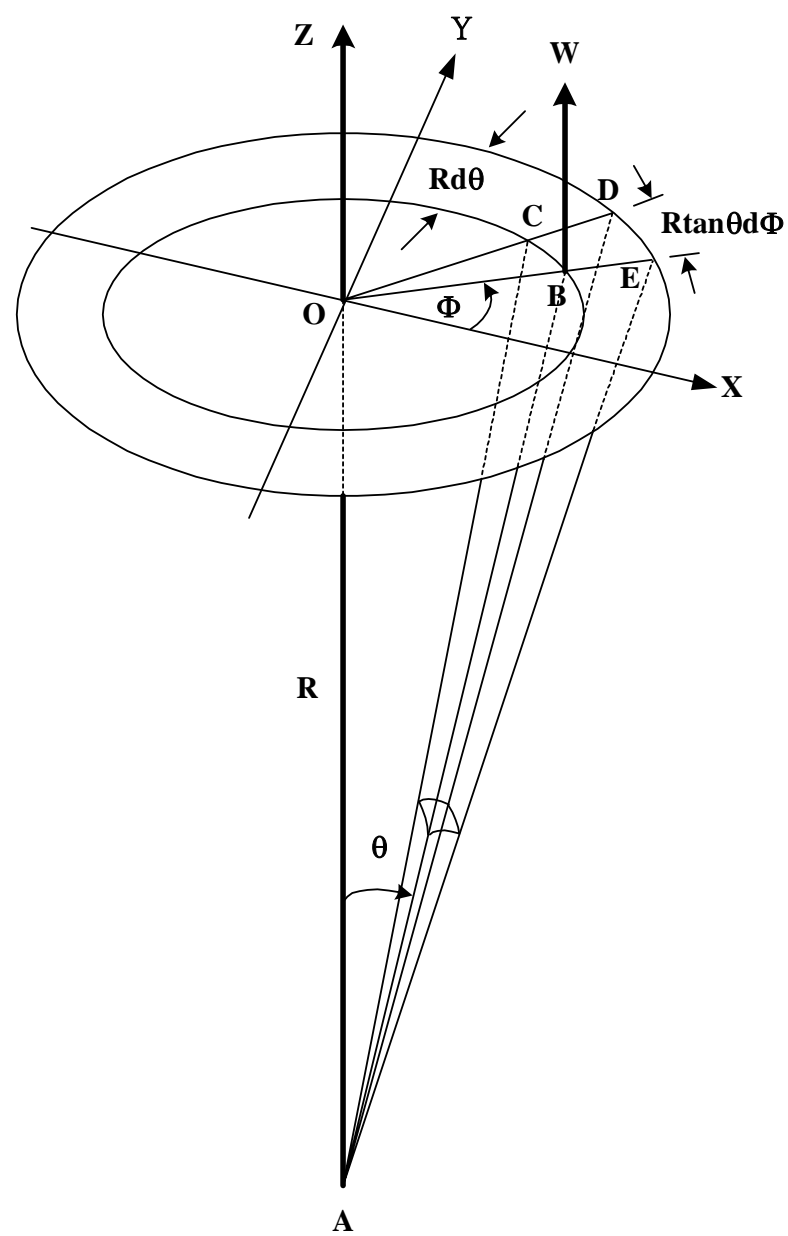

Fig. 1. Geometric relationship between vertically directed antenna beam and vertical wind for the derivation of beam broadening spectrum caused by vertical wind velocity $W$.

Note that $\sigma$ in Eq. (5) is related to the half-power beam width $\theta_{1 / 2}$ of a one-way antenna beam as follows:

$\sigma=\frac{R \theta_{1 / 2}}{2 \sqrt{2 \ln 2}}$

Substituting Eq. (2)-Eq. (6) into Eq. (1), we have

$S_{V}(f) d f=\int_{0}^{2 \pi} G_{0} e^{-(R \tan \theta)^{2} / \sigma^{2}} R^{2} \tan \theta d \Phi d \theta$,

where $G_{0}=g_{0} \Pi_{0}$ and $S_{V}(f)$ is the beam broadening spectral density induced by a uniform vertical wind in a vertically pointed radar beam. Note that the integration in Eq. (7) is performed only along $\Phi$ such that the connection between the power $S_{V}(f) d f$ in the given interval $f$ to $f+d f$ and that in the radar beam in an annulus with infinitesimal area $2 \pi R^{2} \tan \theta d \theta$ in the corresponding angular interval $\theta-\theta+\mathrm{d} \theta$, as obtained from Eq. (4), can be established. Assume further that the antenna beam width is so small that the approximation $\tan \theta \cong \sin \theta \cong \theta$ is valid. As a result, Eq. (7) reduces to

$S_{V}(f) d f=c e^{-(R \sin \theta)^{2} / \sigma^{2}} \theta d \theta$, 


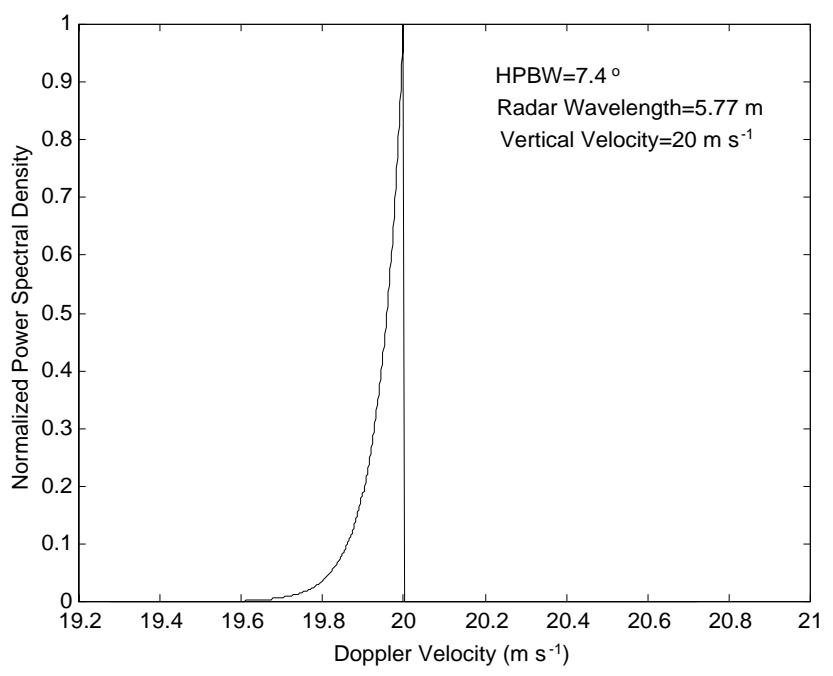

Fig. 2. Example of normalized beam broadening spectrum caused solely by vertical wind, in which a radar wavelength of $5.77 \mathrm{~m}$ (corresponding to $52 \mathrm{MHz}$ ), radar beam with half-power beam width of $7.4^{\circ}$ and $20 \mathrm{~m} \mathrm{~s}^{-1}$ vertical wind velocity are employed in the calculation.

where $c\left(=2 \pi \mathrm{C} \Pi_{0}\right)$ is a constant independent of the height $R$. From Fig. 1, the relation between radial velocity $V_{r}$, vertical wind velocity $w$, and zenith angle $\theta$ can be found easily as seen below

$V_{r}=w \cos \theta$

Equation (9) implies that for a uniformly distributed $w$ in the radar volume, the contour of the Doppler velocity in the radar volume is a set of concentric circles with maximum value at zenith direction. This is one of the reasons why the integration of Eq. (7) is performed over the domain $\Phi$ from 0 to $2 \pi$ with respect to a fixed $\theta$. Notice that the vertical velocity here is assumed to be upward; $V_{r}$, therefore, is positive in Eq. (9). Again, under the assumption of small radar beam width, Eq. (9) can be simplified as

$V_{r} \cong w\left(1-\frac{\theta^{2}}{2}\right)$

By employing the relation between radial velocity and Doppler frequency, namely, $V_{r}=-f \lambda / 2$, where $\lambda$ is the radar wavelength, Eq. (10) becomes

$\theta^{2} \cong 2+\frac{f \lambda}{w}$.

Taking the differential on both sides of Eq. (11) and substituting into Eq. (8), we obtain the normalized beam broadening spectrum

$S_{B}(f)=e^{-\left(f+\frac{2 w}{\lambda}\right) / \sigma_{B}^{2}}$,

where $\quad S_{B}(f)=S_{V}(f) / S_{0}, \quad S_{0}=c \lambda /(2|w|), \quad$ and $\sigma_{B}^{2}=|w| \theta_{1 / 2}^{2} /(8 \lambda \ln 2)$. Because the direction of the vertical wind velocity $w$ determines the sign of the Doppler frequency, there is a limitation imposed on the range of $f$ in Eq. (12), namely, $0 \leq f \leq 2|w| / \lambda$ if $w \leq 0$ or $-2 w / \lambda g$ g, if $w \geq 0$. It should be noted that, except for the procedure mentioned above, there is another way to evaluate the beam broadening spectrum. One can define a spectrum of the velocity variations, which, in turn, is reflected in the measured Doppler frequency spectrum. This is the integral over the product of gain (antenna pattern as a function of zenith and azimuth angle) and the scalar product of the three-dimensional vector of atmospheric velocity and the unit radial direction vector. It also yields all information for any sets of three dimensional wind vectors (and fluctuating cross sections as well), and can also be applied for oblique antenna beams as well. However, for this purpose, one has to first develop the analytic relation between the Doppler frequency and the theta and phi in the illuminating region for the integral. Clearly, it is not an easy task, especially when the oblique radar beam and the three-dimensional wind field are considered (Chu, 2002).

It is evident from Eq. (12) that the pattern of the beam broadening spectrum induced by vertical wind is in exponential form, rather than Gaussian form. In addition, Eq. (12) also indicates that true vertical velocity corresponds to the peak, and not to the first moment (or mean Doppler frequency shift) of $S_{B}(f)$. Figure 2 shows an example of normalized beam broadening spectrum caused by vertical wind only, in which a radar wavelength of $5.77 \mathrm{~m}$ (corresponding to $52 \mathrm{MHz}$ ), radar beam with half-power beam width of $7.4^{\circ}$ and $20 \mathrm{~m} \mathrm{~s}^{-1}$ vertical wind velocity upward are employed in the calculation. As shown, $S_{B}(f)$ following an exponential function with an abrupt discontinuity at Doppler velocity of $-2 w / \lambda$ has a tail declining toward the end of zero Doppler velocity.

It is not difficult to evaluate the mean Doppler frequency $f_{m}$ of $S_{B}(f)$ by performing the following integration

$f_{m}=\frac{\int_{-2 w / \lambda}^{0} f S_{B}(f) d f}{P}$,

where $w$ is assumed to be positive (upward) and $P$ is the zero moment of $S_{B}(f)$ and is defined as

$P=\int_{-2 w / \lambda}^{0} S_{B}(f) d f$.

After performing the integrations, the analytical expression of $f_{m}$ is obtained below

$f_{m}=\frac{|w| \sigma_{b}^{2}}{\lambda}-\frac{2 w / \lambda}{1-e^{-2 / \sigma_{b}^{2}}}$.

Observably, estimated vertical wind velocity $V_{m}\left(=-f_{m} \lambda / 2\right)$ in accordance with Eq. (15), will be different from the actual. Figure 3 presents estimation error $\Delta w$ in $w$ varied with half-power beam width $\theta_{1 / 2}$, where $\Delta w$ is defined as $\left(V_{m}-w\right) / w^{*} 100 \%$. It shows that $\Delta w$ independent of $w$ varies with $\theta_{1 / 2}$ in a parabolic pattern. This result can be readily seen from Eq. (15), if $\theta_{1 / 2}$ is so small (on the order of a few degrees) that approximation $\exp \left(-2 / \sigma_{b}^{2}\right) \sim 0$ is valid. 


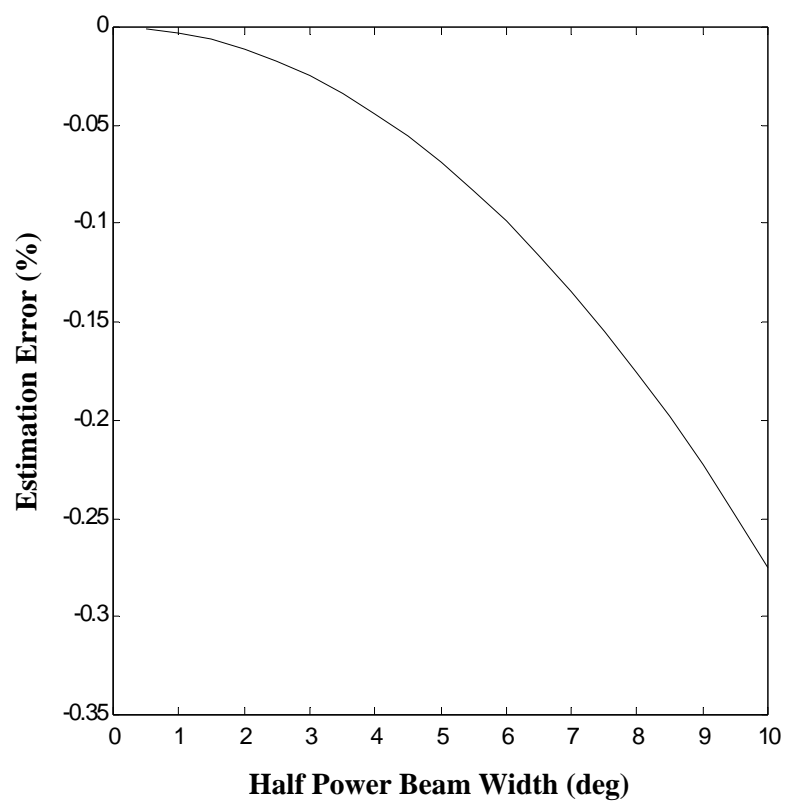

Fig. 3. Estimation error in the estimation of the vertical wind velocity in terms of a first moment of theoretical Doppler spectrum induced by vertical wind, in which the estimation error in unit of percentage is defined as $\left(V_{m}-w\right) / w$, where $V_{m}$ is the mean vertical wind velocity deduced from a first moment of the beam broadening spectrum and $w$ is the true vertical wind velocity.

Overall, the estimation error is minor and insignificant, less than $0.25 \%$ for a typical case.

In an analogous manner, the analytical expression of the width $\sigma_{V}$ of the Doppler spectrum $S_{B}(f)$ due to vertical wind can be evaluated by performing the second moment of $S_{B}(f)$ normalized by $P$, that is,

$\sigma_{V}^{2}=\frac{\int_{-2 w / \lambda}^{0}\left(f-f_{m}\right)^{2} S_{B}(f) d f}{P}$.

After carrying out the mathematical manipulations, we have

$\sigma_{V}^{2}=\left(\frac{|w| \sigma_{b}^{2}}{\lambda}\right)^{2}-\frac{4(w / \lambda)^{2} \exp \left(-2 / \sigma_{b}^{2}\right)}{\left(1-\exp \left(-2 / \sigma_{b}^{2}\right)\right)^{2}}$.

For a radar beam with a few degrees width, because $\exp \left(-2 / \sigma_{b}^{2}\right)$ is exceedingly small, Eq. (17) can be well approximate to be

$\sigma_{V}=\frac{0.18|w|}{\lambda} \theta_{1 / 2}^{2}$

It is evident from Eq. (18) that the beam broadening spectral width caused by vertical wind is proportional to the square of the half-power beam width $\theta_{1 / 2}$, rather than the linear dependence as is the case due to horizontal wind. In order to examine the applicability of Eq. (18), a comparison between spectral widths computed from the approximate Eq. (18) and the second moment of Eq. (12) is made and the results are presented in Fig. 4. As indicated, an excellent agreement between them is seen, validating the applicability of Eq. (18).

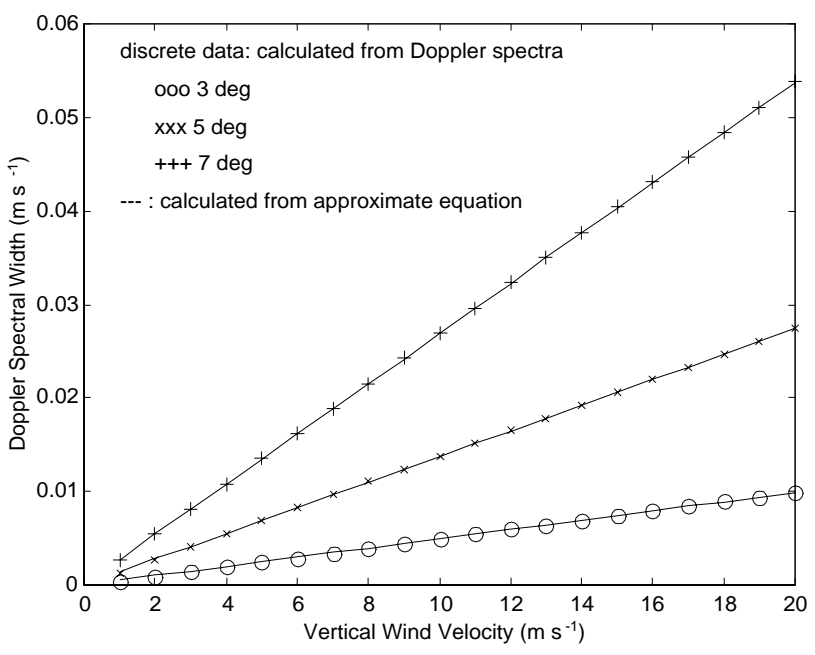

Fig. 4. Comparison between spectral widths calculated from the approximate Eq. (18) (straight lines) and the width of theoretical Doppler spectrum (discrete data point) defined by Eq. (12). It is noteworthy that the magnitude of the spectral width shown in the plot is defined as twice the normalized second moment of Doppler spectrum.

Figure 4 shows that the Doppler spectral width broadened by vertical wind is very small, generally within a few $\mathrm{cm} \mathrm{s}^{-1}$ for the typical cases. However, we will show in the next section that in the presence of horizontal wind vertical wind, plays a crucial role in governing the shape and the width of the beam broadening spectrum for a vertical radar beam. In addition, with the transformation of vertical and wind velocities into along- and cross-radar-beam wind velocities, we will show in Sect. 4 that beam broadening spectral width of an oblique beam may be as narrow as that of the vertical beam, if the magnitudes of the vertical and horizontal wind vectors are at the specific values such that the composite cross-radar-beam wind velocity is negligibly small and the along-radar-beam wind velocity is large. Detailed discussions and mathematical manipulation on the transformation of wind velocities will be given in Sects. 3 and 4.

\section{Doppler spectrum induced by horizontal and vertical winds}

Conventionally, the role that the vertical wind played in characterizing the Doppler spectrum is thought to only shift the mean Doppler velocity of the spectrum and have no contribution to the spectral width. A Gaussian spectrum $\mathrm{S}_{M}(\mathrm{f})$ was generally employed to the model beam broadening spectrum induced by uniform vertical and horizontal winds in the following form (Woodman and Chu, 1989):

$S_{M}(f)=S_{2} e^{-\left(f+\frac{2 U}{\lambda} \cos \zeta \sin \delta=\frac{2 w}{\lambda} \cos \delta\right)^{2} / 2 \sigma^{2}(\delta)}$,

where $\delta$ is the zenith angle of radar beam, $\zeta$ is the azimuth angle of the horizontal wind with respect to the radar beam axis, $\sigma(\delta)\left(=\sigma_{B} \cos \delta\right)$ is the beam broadening width at zenith angle 

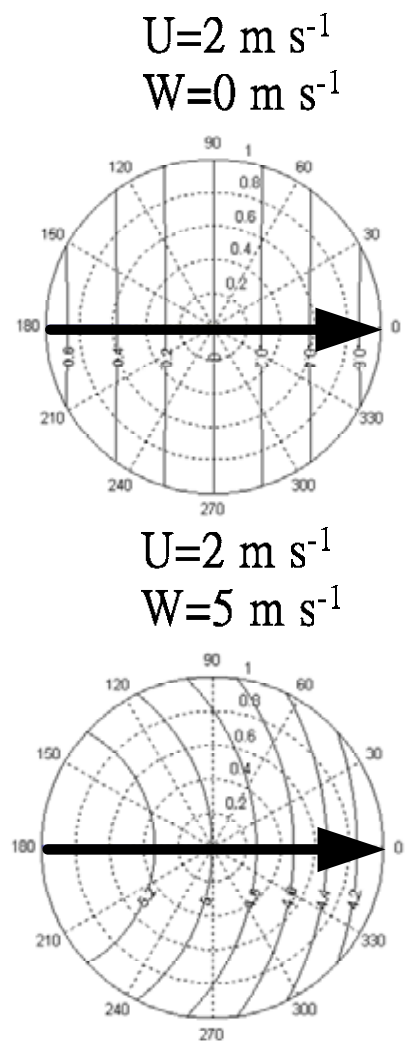

$\mathrm{U}=2 \mathrm{~m} \mathrm{~s}^{-1}$ $\mathrm{W}=10 \mathrm{~m} \mathrm{~s}^{-1}$

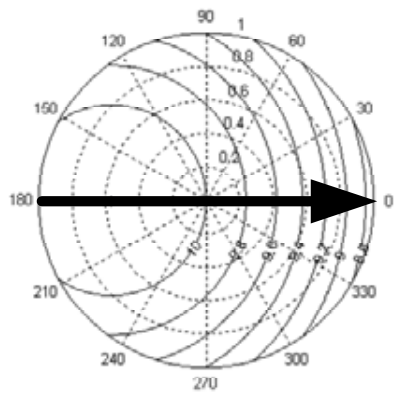

Fig. 5. Comparison of contours of radial velocity for the different ratios of vertical wind velocity to horizontal wind velocity, in which a bold arrow in each panel shows the horizontal wind direction.

$\delta$, and $\sigma_{B}$ as the function of horizontal wind velocity $U$ and half-power beam width $\theta_{1 / 2}$ is defined as $U \theta_{1 / 2} /\left(2(\ln 2)^{1 / 2} \lambda\right)$. It should be noted that in Eq. (19) the wind shear effect and other broadening factors are not considered, although they may play some roles in the width of $S_{M}(f)$ (Nastrom, 1997). In the following, with the help of numerical simulation, we will show that in the condition of the large ratio of $w$ to $U$, the vertical wind contributes significantly to the spectral width, suggesting an inaccurate description of the beam broadening spectral width in terms of Eq. (19).

Although the analytical expression of the beam broadening spectrum for a vertically pointed antenna beam induced by vertical wind solely is derived as shown in Eq. (12), it is very difficult to formulate the analytical expression of the beam broadening spectrum induced by horizontal in wind combination with vertical winds. This is because when the ratio of vertical to horizontal wind velocities is not negligibly small, the contour of radial velocity is curved and the integration of radar power along the curved radial velocity contour will be very difficult to perform (Sloss and Atlas, 1968). In order to solve this difficulty, a numerical simulation technique is employed to achieve the corresponding beam broadening spectrum.

To begin with, the radial velocity at the point with angular position $(\theta, \Phi)$ on the cross section of a vertically pointed radar beam can be expressed below as:

$V_{r}=U \cos \Phi \sin \theta+w \cos \theta$,

where $U$ and $w$ are, respectively, horizontal and vertical wind. Notice that in Eq. (20) the reference axis for azimuth angle $\Phi$ is taken as the direction of the horizontal wind. In order to realize the effects of $w$ and $U$ on the configuration of the radial velocity contour in the resolution volume, we calculate the magnitude of $\mathrm{V}_{r}$ for different $w$ and $U$ and plot their contour on the cross section of the radar beam. Figure 5 demonstrates three examples of the contours of radial velocity (solid curves) for a vertical radar beam, in which $2 \mathrm{~m} \mathrm{~s}^{-1}$ of horizontal wind velocity and 0,5 , and $10 \mathrm{~m} \mathrm{~s}^{-1}$ vertical wind velocities are employed in the calculations. The concentric circles marked with dashed curves in Fig. 5 are the contours of zenith angle $\theta$, which have been normalized by a factor of $20^{\circ}$, and the bold arrow represents the vector of horizontal wind and the numbers attached to the contours represent the corresponding radial velocity. It is clear from Fig. 5 that the configuration of the radial velocity contours vary with the relative magnitude of the vertical wind velocity compared to the horizontal wind velocity. They are well approximate to a straight line when $w$ is relatively small, and they are more like a concentric circle centered at the maximum radial velocity when $w$ is relatively large. The detailed characteristics of the radial velocity contour will be investigated and discussed in another paper and will not be given here.

By specifying the antenna beam pattern, and vertical and horizontal wind velocities, where the horizontal wind direction is adopted as the reference axis for the measurement of azimuth angle $\Phi$, we can obtain the beam broadening spectrum by numerically integrating all radar power along the radial velocity contour in the resolution volume. Figure 6 shows the numerical beam broadening spectrum (solid curve) and the conventional Gaussian spectrum (dashed curve), calculated in accordance with Eq. (19), in which a vertically directed radar beam with $5^{\circ}$-half-power beam width, $5.77-\mathrm{m}$ radar wavelength, $1 \mathrm{~m} \mathrm{~s}^{-1}$ horizontal and $-20 \mathrm{~m} \mathrm{~s}^{-1}$ vertical wind velocities (downward) are employed for the computation. As indicated in Fig. 6, the shape of the beam broadening spectrum deviates significantly from that of the Gaussian spectrum. The asymmetry of the beam broadening spectrum leads to the discrepancies of its mean Doppler velocity and 


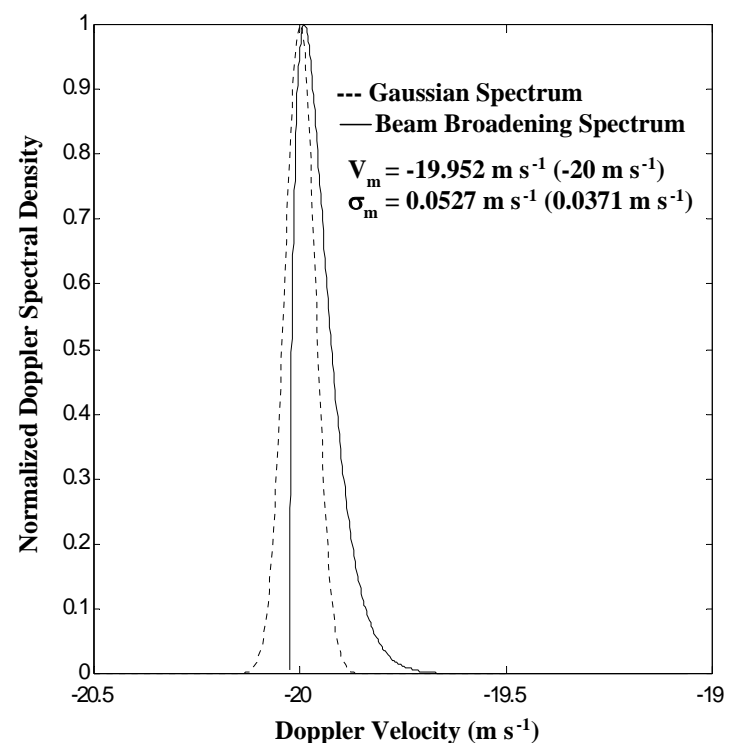

Fig. 6. Comparison between beam broadening spectrum (solid curve) obtained by numerically integrating radar power along the contours of radial velocity and a Gaussian spectrum (dashed curve) with a spectral width of $0.0371 \mathrm{~m} \mathrm{~s}^{-1}$ (corresponding to $1 \mathrm{~m} \mathrm{~s}^{-1}$ of horizontal wind velocity for a $52-\mathrm{MHz}$ antenna beam with $5^{\circ}$ width), in which $V_{m}$ and $\sigma_{m}$ are, respectively, estimated mean Doppler velocity and spectral width of the exact beam broadening spectrum by means of the moment method.

spectral width from the true vertical velocity and Gaussian spectral width. The calculation shows that for the present case, the estimation error in vertical wind velocity is very minor and only about $0.24 \%$, while the difference in the spectral widths is significant and can be as large as $42.1 \%$. An extended survey on the relative discrepancies (in percentage) in the estimations of vertical wind velocity and spectral width is made and the results are presented in Figs. 7 and 8 , where the relative error is defined as $\left(V_{m}-w\right) / w * 100 \%$ for vertical wind velocity and the relative discrepancy is defined as $\left(\sigma_{m}-\sigma_{B}\right) / \sigma_{B} * 100 \%$ for spectral width, and $\mathrm{V}_{m}$ and $\sigma_{m}$ are, respectively, the first and second moments of the numerical beam broadening spectrum and $\sigma_{B}$ is defined as $U \theta_{1 / 2} /\left(2(\ln 2)^{1 / 2} \lambda\right)$. As shown in Fig. 7 , the relative error in vertical wind velocity is a function of antenna beam width and the ratio of vertical $w$ to horizontal $U$ wind speeds and their mathematical relation can be described by a linear equation, which is employed to best fit to the data, as below:

Error $=\alpha+\beta \times(w / U)(\%)$

where $\alpha$ and $\beta$ values corresponding to $3^{\circ}, 5^{\circ}$ and $7^{\circ}$ half-power beam widths are, respectively, $-0.098,-0.273$, -0.535 and $0.00013,0.0008,0.0029$. Analysis further reveals that the $\alpha$ value is related to the half-power beam width $\theta_{1 / 2}$ by a power of 2 , namely, $\alpha=c \theta_{1 / 2}^{2}$, where $c$ is equal to -0.0109 and $\theta_{1 / 2}$ is in units of degree. However, no relation between $\beta$ and $\theta_{1 / 2}$ is found. Figure 8 shows the relative discrepancy $\Delta \sigma$ between $\sigma_{m}$ and $\sigma_{B}$. In a similar manner,

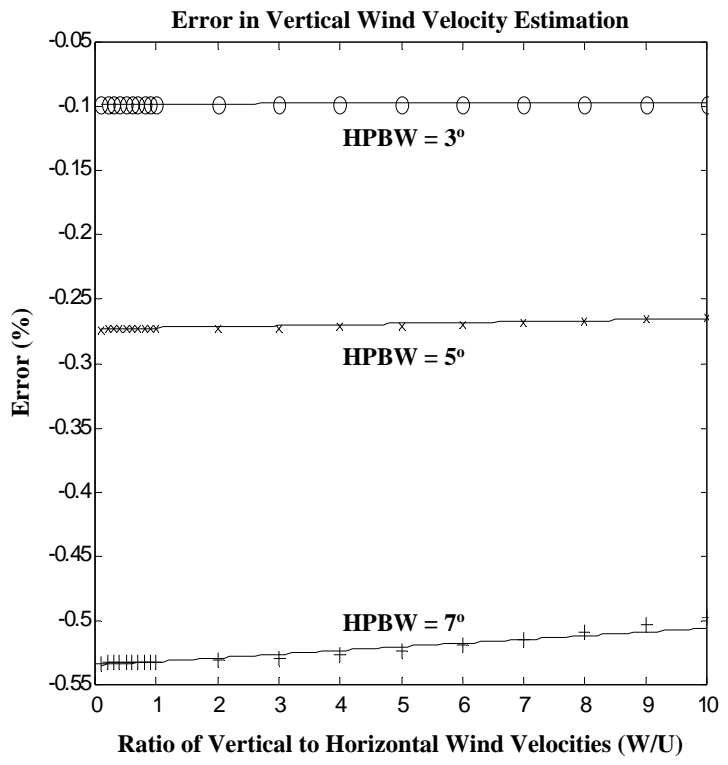

Fig. 7. Estimation error in the vertical wind velocity calculated from the first moment method of the beam broadening spectrum caused by vertical and horizontal winds.

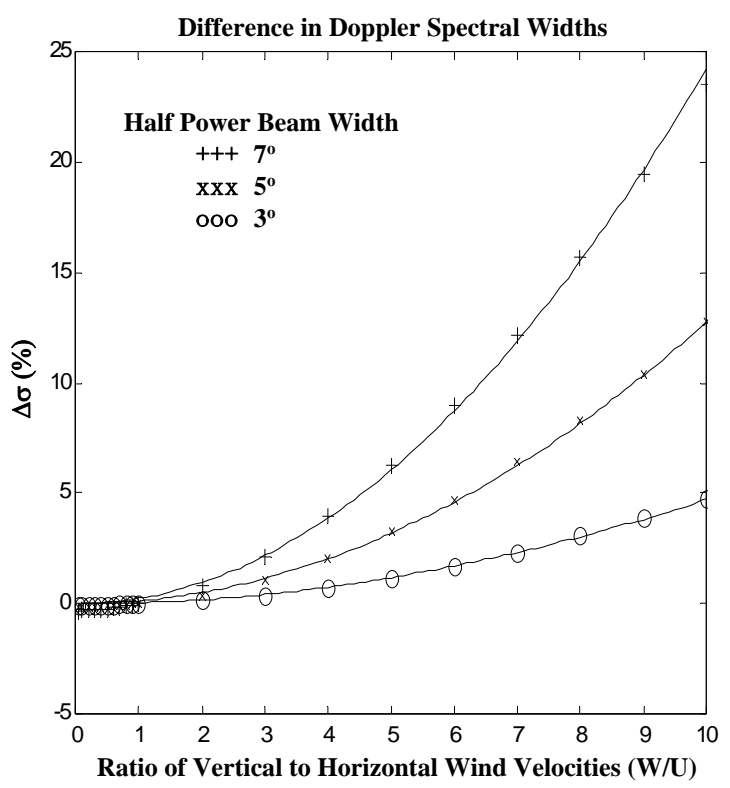

Fig. 8. Differences between spectral widths estimated from Doppler spectrum subject to horizontal wind in combination with vertical winds and a Gaussian beam broadening spectrum caused by horizontal wind only.

we find that $\Delta \sigma$ is also a function of the ratio of vertical to horizontal wind speeds and their relationship can be well described by a parabolic equation expressed below as:

$\Delta \sigma=\gamma \times(w / U)^{2}(\%)$,

where $\gamma$ values corresponding to $\theta_{1 / 2} 3^{\circ}, 5^{\circ}$ and $7^{\circ}$ are, respectively, $0.0473,0.1275$ and 0.2423 . Again, $\gamma$ is related to $\theta_{1 / 2}$ by a power of 2 and can be expressed as $\gamma=\mathrm{G} \theta_{1 / 2}^{2}$, 


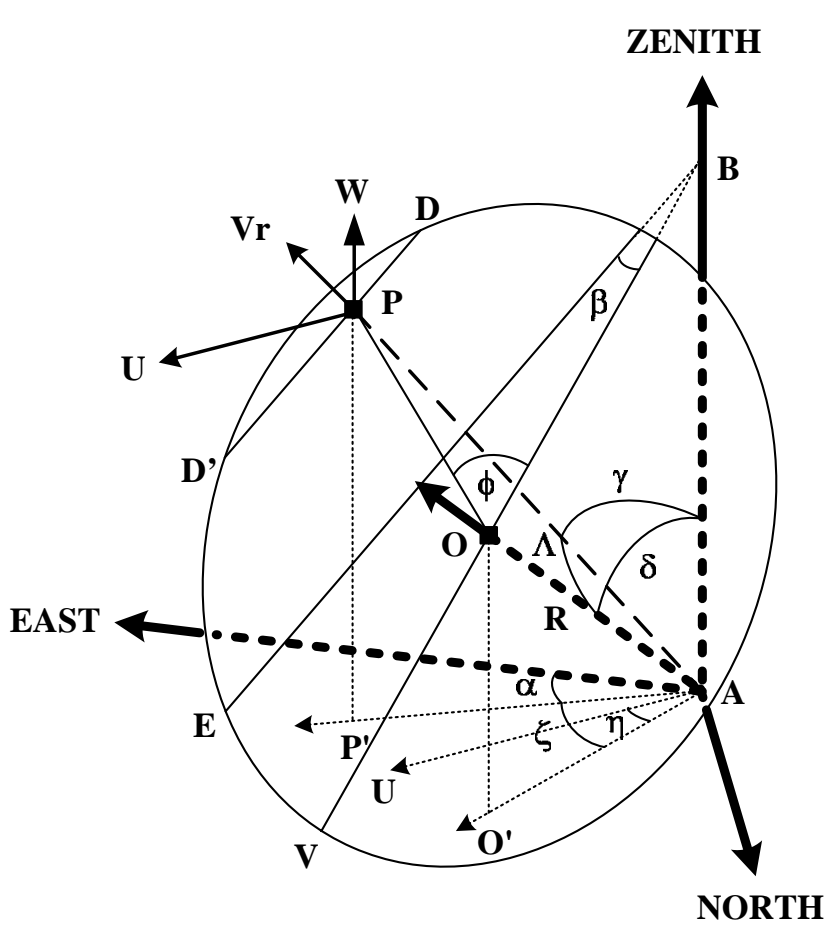

Fig. 9. Schematic configuration showing the geometric relations between coordinate, pointing vector $\overrightarrow{A O}$ of an oblique antenna beam with zenith angle $\delta$, horizontal $U$ and vertical $W$ wind vectors, and the angular position of selected point $P$ on the cross section of the oblique antenna beam pattern, where the coordinate of the point $P$ is defined by cone angles $\Lambda$ and azimuth angles $\phi$ with respect to the boresight of oblique antenna beam. For details of the other symbols in the plot, see text.

where the magnitude of $\mathrm{G}$ is about 0.005 and $\theta_{1 / 2}$ is in units of degree. From Eq. (22), we see that $\Delta \sigma$ will increase dramatically with the increases in $w / U$ and $\theta_{1 / 2}$, suggesting that the vertical wind velocity effect on the width of the Doppler radar spectrum should be taken into consideration in the condition of large $w / U$ and broad antenna beam. In addition, in light of a perfect agreement between the calculated data and the parabolic equation, as shown in Fig. 8, $\sigma_{m}$ can be achieved in terms of $\sigma_{B}$ in accordance with Eq. (22) without the use of the numerical integration to construct the corresponding beam broadening spectrum.

\section{Transformation of wind velocity}

Although the results obtained in Sects. 2 and 3 are based on the vertically pointed radar beam, we will show in this section that they can be applied to the oblique radar beam through the transformation of vertical and horizontal wind velocities into along- and cross-radar beam wind velocities defined on the cross section of oblique radar beam. Figure 9 presents a schematic diagram showing the geometry of the cross section of an oblique antenna beam with tilted angle $\delta$ at slant range $R$, in which $\overrightarrow{A O}$ is the pointing vector of the antenna beam axis, $\overrightarrow{B V}$ is the projection of the vector
$\overrightarrow{A O}$ on the cross section of the oblique beam, $\overrightarrow{A P}$ is the vector connecting antenna $A$ and an arbitrary point $P$ on the oblique cross section, $\overrightarrow{B E}$ is the intersection of the oblique plane and the vertical plane comprising the horizontal wind vector through vertical axis $A B, D D^{\prime}$ is the line through the point $P$ parallel to $\overrightarrow{B E}$, the angular coordinate of the selected point $P$ is $(\Lambda, \phi)$ and $\Lambda$ is called a cone angle relative to vector $\overrightarrow{A O}$ and $\phi$ is termed as a clock angle with respect to axis $\overrightarrow{B V}$. Note that the vector $\overrightarrow{A O}$ is normal to the oblique plane at point $O$ such that the plane is tilted with vertical axis at the angle of $\pi / 2-\delta$. Referring to Fig. 9 , the radial velocity $\mathrm{V}_{r}$ at point $P$, subjected to a uniform background wind, can be formulated as:

$V_{r}=u \times \cos \alpha \times \sin \gamma+v \times \sin \alpha \times \sin \gamma+w \times \cos \gamma$

where $u$ and $v$ are, respectively, the eastward and northward components of the horizontal wind, $w$ is the vertical wind velocity, $\alpha$ is the angle between the axis toward due east and vector $\overrightarrow{A P^{\prime}}$ which is the projection of vector $\overrightarrow{A P}$ on horizontal plane, and $\gamma$ is zenith angle of vector $\overrightarrow{A P}$. Referring to Fig. 9, by introducing angles $\zeta$ and $\eta$, Eq. (23) can be expressed below as:

$V_{r}=U \cos (\zeta-\eta) \times \sin \gamma+w \times \cos \gamma$,

where $U\left(=\sqrt{u^{2}+v^{2}}\right)$ is the horizontal wind velocity, $\eta$ is the angle between horizontal wind vector and $\overrightarrow{A O^{\prime}}$, and $\zeta$ is the angle between $\overrightarrow{A O^{\prime}}$ and $\overrightarrow{A P^{\prime}}$, where $O^{\prime}$ and $P^{\prime}$ are, respectively, the projections of the points $O$ and $P$ on the ground. From Fig. 9, the following identities can be obtained:

$\cos \gamma=\cos \delta \cos \Lambda+\sin \delta \sin \Lambda \cos \phi$,

$\sin \zeta \sin \gamma=\sin \Lambda \sin \phi$,

$\cos \zeta \sin \gamma=\sin \delta \cos \Lambda-\cos \delta \sin \Lambda \cos \phi$

Substituting Eq. (25), Eq. (26), and Eq. (27) into Eq. (24), we have the radial velocity at point $P$ as below:

$V_{r}=U \cos \eta(\sin \delta \cos \Lambda-\cos \delta \sin \Lambda \cos \phi)$

. $U \sin \eta \sin \Lambda \sin \phi+w(\cos \delta \cos \Lambda+\sin \delta \sin \Lambda \cos \phi)$

To proceed, we decompose the total wind vector $V$, which is determined by the given wind vectors $w$ and $U$, into two mutually orthogonal components $V_{\perp}$ and $V_{\|}$, where $V_{\perp}$ is the component of $V$ along the beam axis $\overrightarrow{A O}$ and is called an along-radar-beam wind and $V_{\|}$is the one lying on the oblique plane and called a cross-radar-beam wind perpendicular to $\overrightarrow{A O}$. After tedious mathematical manipulation, Eq. (28) can be transformed into the following expression:

$V_{r}=V_{\|}^{\perp} \sin (\phi+\beta) \sin \Lambda+V_{\|}^{\|} \cos (\phi+\beta) \sin \Lambda+V_{\perp} \cos \Lambda$

where $\phi$ is the clock angle of point $P$ with respect to line $\overrightarrow{B V}, \beta$ is the angle between $\overrightarrow{B E}$ and $\overrightarrow{B V}, V_{\|}^{\perp}$ and $V_{\|}^{\|}$are, respectively, the components of the cross-radar-beam wind 
$V_{\|}$projected to the line $C C^{\prime}$ and to the line $B B^{\prime}$. Note that line $C C^{\prime}$ is the line parallel to $\overrightarrow{B E}$ through the point $O$ on the oblique plane and line $B B^{\prime}$ is the one normal to $C C^{\prime}$ on the oblique plane, as shown in Fig. 10. The expressions of $V_{\|}^{\perp}$ and $V_{\|}^{\|}$can be formulated in terms of $w, U, \delta, \beta$, and $\eta$ as below

$V_{\|}{ }_{\|}=w \sin \delta \sin \beta-U \cos \eta \cos \delta \sin \beta+U \sin \eta \cos \beta$

$V_{\|}^{\|}=w \sin \delta \cos \beta-U \cos \eta \cos \delta \cos \beta-U \sin \eta \sin \beta$

and the expression of $\mathrm{V}_{\perp}$ can be expressed as

$V_{\perp}=U \cos \eta \sin \delta+w \cos \delta$.

Inspecting Eq. (30)-Eq. (32) shows that for the case of background wind uniformly distributed in the resolution volume $V_{\|}^{\perp}, V_{\|}^{\|}$and $V_{\perp}$ are independent of cone angle $\theta$ and azimuth angle $\phi$ on the oblique plane. By introducing velocity $V_{\|}$and angle $\Theta$, Eq. (29) can be further rewritten as:

$V_{r}=V_{||} \cos \Psi \sin \Lambda+V_{\perp} \cos \Lambda$,

where $\quad \Psi=\phi+\beta-\Theta, \quad V_{\|}=\sqrt{\left(V_{\|}^{\perp}\right)^{2}+\left(V_{\|}^{\|}\right)^{2}}, \quad$ and $\Theta=\tan ^{-1}\left(V_{\|}^{\perp} / V_{\|}^{\|}\right)$. Obviously, Eq. (33), developed for the oblique radar beam, is exactly identical to Eq. (20) for the vertically directed radar beam, in which the crossradar-beam wind $V_{\|}$and the along-radar-beam wind $V_{\perp}$ on the oblique plane are, respectively, equivalent to the horizontal wind $U$ and the vertical wind $\mathrm{w}$ on the horizontal plane of a vertical radar beam, and $\Psi$ measured with respect to the line $C C^{\prime}$ on the oblique plane is equivalent to $\Phi$ defined with respect to the horizontal wind direction on cross section of vertical radar beam. In light of the equivalence in the two, the theoretical results obtained in Sects. 2 and 3 in the condition of the vertically directed radar beam can be directly applied to the case of the oblique beam by simply substituting $V_{\perp}$ and $V_{\|}$on the oblique plane for $w$ and $U$ on the horizontal plane, respectively. Inspecting expressions Eq. (30)-Eq. (33) implies that the behavior of the resultant Doppler spectrum will depend heavily on the direction and speed of the background wind and tilt angle of the radar beam. It follows that the changes in $U, w$ and $\beta$ will lead to the changes in the shape and width of the corresponding Doppler spectrum. Under the special conditions of $\Psi=90^{\circ}$ or $V_{\|}=0, V_{r}$ will be governed by $V_{\perp}$ only and the shape of corresponding Doppler spectrum will be in the exponential form with exceedingly narrow spectral width defined by Eq. (17) or Eq. (18), in which $w$ is replaced by $V_{\perp}$. As a result, for an oblique radar beam the asymmetrical Doppler spectrum with a tremendously narrow width (as small as a few $\mathrm{cm} \mathrm{s}^{-1}$ ) may be expected, irrespective of the presence of a fairly large horizontal wind velocity. Obviously, in this case the Gaussian spectrum, as shown in Eq. (19), is inappropriate to model the spectrum and remarkable error in the estimation of spectral width in terms of $\sigma_{B}$ will be induced.

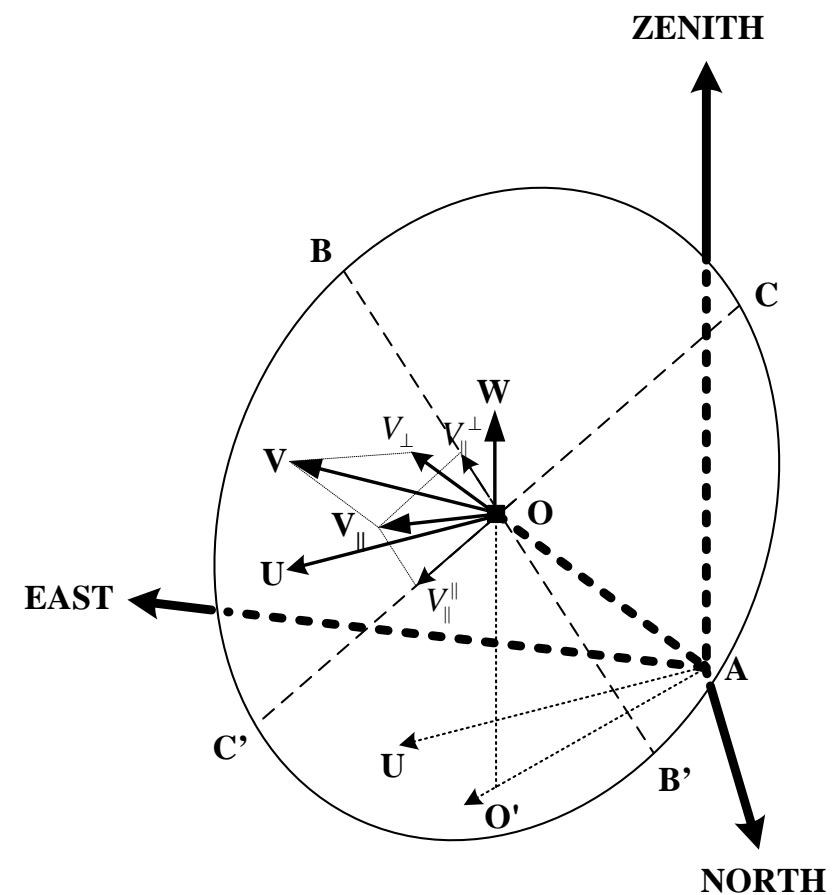

Fig. 10. Geometric relations between the wind vectors $w, U, V$, $V_{\perp}, V_{\|}, V_{\|}^{\perp}$, and $V_{\|}^{\|}$, in which $C C^{\prime}$ is the line through point $O$ parallel to $\overrightarrow{B E}$ as shown in Fig. 9 and $B B^{\prime}$ is the one normal to $C C^{\prime}$ on the oblique plane.

\section{Discussion}

One of the advantages of Doppler radar in remote sensing of the atmosphere is the capability of measuring turbulence strength aloft. The information of turbulence embedded in the radar returns from refractivity fluctuations can be retrieved either from backscattered power or from observed Doppler spectral width (Cohn, 1995). For the spectral width method, the uses of radar beams pointed vertically or obliquely are common in the Doppler radar community (Kurosaki et al., 1996; Nastrom and Eaton, 1997). With this method, the turbulence strength is estimated by removing the contributions of beam broadening, wind shear broadening, and gravity wave broadening from the observed Doppler spectral width of the oblique beam in accordance with the following expression (Nastrom and Eaton, 1997)

$\sigma_{t}^{2}=\sigma_{o b s}^{2}-\sigma_{\text {Beam }+ \text { shear }}^{2}-\sigma_{\text {wave }}^{2}$.

It is noteworthy that for the vertical radar beam, although shear and wave broadening effects can be reasonably ignored, the effect of specular reflection from thin layers in the illuminating volume should be considered for the radar operating at VHF band (Rottger and Larsen, 1990). Although Eq. (34) has been used extensively in the radar community to remove non-turbulence broadening spectral width, a number of factors that may contribute significantly to observed Doppler spectral width are not included. For example, Chu (2002) shows that the horizontal wind direction relative 
to the obliquely directed radar beam direction can cause an estimation error in the beam broadening spectral width as large as more than $15 \%$. Our results presented in Sects. 3 and 4 indicate that the ratio of $w$ to $U$ for vertical beam or the ratio of $V_{\perp}$ to $V_{\|}$for the oblique beam are the crucial factors influencing the Doppler spectral width. Quantitative computation reveals that for the vertical radar beam the spectral broadening induced by vertical and horizontal winds will be greater by $11 \%$ or more than beam broadening due to horizontal wind only, where $6^{\circ}$ radar beam width and $U=1.25 \mathrm{~m} \mathrm{~s}^{-1}$ and $w=10 \mathrm{~m} \mathrm{~s}^{-1}$ are assumed in the computation. However, for an oblique radar beam with a tilt angle of $15^{\circ}$ and the same beam width and wind velocities, the former will be substantially greater than the latter by more than $24 \%$, where the assumption that the horizontal wind direction is parallel to the azimuth angle of the antenna beam axis is made. This result strongly suggests that the effects of perpendicular wind $V_{\perp}$ for the oblique beam (or vertical wind velocity for the vertical beam) on the beam broadening spectral width should be taken into consideration for the accurate retrieval of the turbulence parameter from the observed Doppler spectral width.

Radar experiments conducted at VHF band indicate that it is not uncommon that the observed Doppler spectral width for an oblique beam is less than the combination of the beam broadening, shear broadening and gravity wave broadening (Nastrom, 1997; Nastrom and Eaton, 1997). One of the causes for this phenomenon is attributed to the vertical variation of the horizontal wind velocity in the illuminating volume. Nastrom (1997) shows that vertical wind shear with a positive sign (i.e. horizontal wind velocity increases with height) will result in a negative contribution to the spectral broadening, leading to the reduction of Doppler spectral width. However, for a uniform background wind (i.e. no wind shear effect), the theoretical results presented in Sects. 3 and 4 predict that the beam broadening spectrum with a tremendously narrow width can occur under the conditions of $\Psi=90^{\circ}$, or $\delta=90^{\circ}$ together with $\eta=0$ and $w=0$, or $\Gamma=\delta$, in combination with $\eta=\beta=0$, where $\Gamma$ is defined as $\cot ^{-1}(w / U)$. In these cases, the contribution of the crossradar-beam wind $V_{\|}$to radial velocity $V r$ will vanish. As a result, the corresponding Doppler spectrum will be governed by the along-radar-beam wind $V_{\perp}$ in accordance with Eq. (12) and a tremendously narrow spectral width is then expected.

In addition to the clear-air turbulence strength, the theory developed in the article may also apply to the precipitation Doppler spectrum observed by obliquely directed Doppler radar. Note that the falling velocity (or Doppler velocity) of a raindrop is the combination of its terminal velocity and vertical wind velocity, and the maximum terminal velocity of a raindrop can be as large as about $10 \mathrm{~m} \mathrm{~s}^{-1}$ (Spilhous, 1948; Gunn and Kinser, 1949; Atlas et al., 1973). Consequently, the falling velocity of raindrop may be much greater than $10 \mathrm{~m} \mathrm{~s}^{-1}$, if the raindrop size is large and the downward vertical wind velocity is intense (Rao et al., 1999). According to the results obtained in Sects. 3 and 4, for the raindrop with a large ratio of falling velocity to horizontal wind velocity observed by a vertical or oblique radar beam, the corresponding precipitation Doppler spectrum will have the breadth greater than that of the conventional Gaussian spectrum broadened by horizontal wind only. As a result, the raindrop distribution will be overestimated if the contribution of the large falling velocity of the raindrop to the beam broadening spectral width is not considered and only the conventional beam broadening spectral width $\sigma_{B}$ is removed from the observed precipitation Doppler spectrum. Therefore, a more complete algorithm is required to develop the estimation of more accurate precipitation information from the observed precipitation Doppler spectrum under the conditions of heavy rain and a weak horizontal wind.

The asymmetrical behavior of the beam broadening spectrum induced by an along-radar-beam wind may also be one of the influential factors responsible for the skewness of type 1 radar spectra in the ionospheric sporadic-E (Es) region in the height range between $90 \mathrm{~km}$ and $150 \mathrm{~km}$. The electron density irregularities responsible for the Es type 1 radar spectra are excited through a two-stream instability and propagate in the direction perpendicular to the magnetic field line (Farley, 1963). Coherent VHF radar has long been shown to be able to effectively detect the echoes from type 1 irregularities by steering the radar beam perpendicular to the magnetic field line (e.g. Kelley, 1989). Es type 1 radar spectra are characterized by a relatively narrow spectral width compared to a fairly large mean Doppler velocity nominally at the speed of an ion-acoustic wave. It is well known that the ion-acoustic wave velocity in the Es region ranges from about 250 to $360 \mathrm{~m} \mathrm{~s}^{-1}$, depending on the latitude and height where type 1 irregularities are generated (e.g. Fejer and Kelley, 1980; Haldoupis et al., 1997). Apparently, exceedingly large drift velocity of type 1 irregularities along the radar beam will not only broaden the width of type 1 radar spectra, but also significantly influence the shape of the radar spectra, producing an asymmetrical shape of the spectra. Although an effective antenna beam pattern (defined as the product of antenna beam pattern and aspect sensitivity) for the measurement of field-aligned Es irregularities is fan-like (i.e. very narrow in elevation and fairly wide in azimuth direction) (Chu and Wang, 1999, Chu and Wang, 2002), the results obtained in this article are still applicable to Es type 1 radar spectra by replacing the original antenna pattern with an effective antenna pattern in Eq. (1), provided that the irregularities fill in the expected echoing region. A detailed discussion on this subject will be given in another paper. In fact, a wealth of observational evidences obtained by various radar experiments show that the radar spectra of type 1 irregularities indeed exhibit a one-sided low frequency wing (e.g. Fejer et al., 1975; Schlegel and Haldoupis, 1994; Chu and Wang, 2002). This kind of asymmetrical shape of the radar spectrum seems to be consistent with that of the example of the spectrum shown in Fig. 6. Therefore, in combination with horizontal drift of the electron density irregularities, the beam broadening effect due to an exceedingly large velocity of the electron density irregularities drifting along the radar beam axis may be 
a plausible cause for the explanation of the asymmetry of $E s$ type 1 radar spectra.

\section{Conclusion}

The analytical expression of beam broadening spectrum for the vertical radar beam induced by vertical wind in the absence of a horizontal wind is derived in this article and its spectral shape is found to be in exponential form. The difference between the mean Doppler velocity of the exponential spectrum and the true vertical wind velocity is the function of the antenna beam width only, while its spectral width is proportional to the product of the vertical wind velocity and the square of the radar half-power beam width. The characteristics of the beam broadening spectrum induced by vertical wind in combination with horizontal winds for the vertical beam are also investigated in this article by using a numerical simulation method, which is very difficult to obtain analytically due to the curved nature of the radial velocity contour. The results reveal that the difference between the mean radial velocity of the beam broadening spectrum and the true vertical wind velocity as a function of the ratio $w / U$ is very small, generally less than $0.6 \%$ for the typical condition. However, its spectral width is greater than that calculated from the modeled Gaussian spectrum broadened by a horizontal wind only, by $15 \%$ for the typical case. Moreover, by employing an empirical parabolic equation proposed in this article, the width of the beam broadening spectrum broadened by the vertical and horizontal winds can be estimated in terms of the beam broadening spectral width caused by the horizontal wind only, without numerically constructing the beam broadening spectrum by integrating the radar power along the curved contour of the radial velocity. A transformation of the vertical and horizontal wind velocities into an along-radar-beam wind $V_{\perp}$ and cross-radar-beam wind velocities $V_{\|}$for an obliquely directed radar beam is developed in this article. One of the advantages of this transformation is that, by incorporating the beam broadening spectral width induced by the horizontal wind, the beam broadening spectral width for the oblique radar beam, under the condition that the contour of the Doppler velocity on the cross section of the radar beam is curved and very difficult to be modeled by mathematical equation, can be computed in accordance with Eq. (22) by simply replacing $w$ and $U$ with $V_{\perp}$ and $V_{\|}$ defined on an oblique plane, respectively. With the wind velocity transformation, under a special condition the oblique Doppler spectrum with exceedingly narrow width (smaller than a few $\mathrm{cm} \mathrm{s}^{-1}$ ) is expected, irrespective of the presence of a large horizontal wind velocity and broad antenna beam width.

Acknowledgements. I would like to thank Hoppe, U.-P. and one of the anonym referees for their helpful and constructive suggestions on this paper. This work was partially supported by National Science Council of the Republic of China (Taiwan) under grant NSC93-2111-M-008-025-AP3 and National Space Program Office under the grant 93-NSPO(B)-RS3-FA07-01.
Topical Editor U.-P. Hoppe thanks two referees for their help in evaluating this paper.

\section{References}

Atlas, D., Srivastava, R. C., and Sekhon, R. S.: Doppler radar characteristics of precipitation at vertical incidence, Rev. Geophys. Space. Sci., 11, 1-35, 1973.

Battan, L. J. and Theiss, J. B.: Observations of vertical motions and particle sizes in a thunderstorm, J. Atmos. Sci., 23, 78-87, 1966.

Brandes, E. A., Vivekanandan, J., Tuttle, J. D., and Kessinger, C. J.: A study of thunderstorm microphysics with multiparameter radar and aircraft observations, Mon. Wea. Rev., 123, 31293143, 1995.

Chu, Y. H.: Beam broadening effect on oblique MST radar Doppler spectrum, J. Atmos. Oceanic Technol., 19, 1955-1967, 2002.

Chu, Y. H., Chao, J. K., Liu, C. H., and Röttger, J.: Aspect sensitivity at tropospheric height measured with vertically pointed antenna beam of the Chung-Li VHF rada, Radio Sci., 25, 539550, 1990.

Chu, Y. H. and Wang, C. Y.: Interferometry investigations of VHF backscatter from plasma irregularity patches in the nighttime $\mathrm{E}$ region using the Chung-Li radar, J. Geophys. Res., 104, 26212631, 1999.

Chu, Y. H. and Wang, C. Y.: Three-dimensional Spatial Structures of Mid-Latitude Type 1 Es Irregularities, J. Geophys. Res., 107(A8), 10.1029/2001JA000215, 2002. 2002.

Cohn, S. A.: Radar measurements of turbulent eddy dissipation rate in the troposphere: A comparison of technique, J. Atmos. Oceanic Technol., 12, 85-95, 1995.

Farley, D. T.: A plasma instability resulting in field-aligned irregularities in the ionosphere, J. Geophys. Res., 68, 6083-6094, 1963.

Fejer, B. G. and Kelley, M. C.: Ionospheric Irregularities, Rev. Geophys., 18, 401-454, 1980.

Fejer, B. G., Farley, D. T., Balsley, B. B., and Woodman, R. F.: Oblique VHF radar spectral studies of the equatorial electrojet, J. Geophys. Res., 80, 1307-1312, 1975.

Gunn, R. and Kinser, G. D.: The terminal velocity of fall for water droplets in stagnant air, J. Meteor., 6, 243-248, 1949.

Haldoupis C., Farley, D. T., and Schlegel, K.: Type-1 echoes from the mid-latitude E-region ionosphere, Ann. Geophys., 15, 908917, 1997,

SRef-ID: 1432-0576/ag/1997-15-908.

Hocking, W. K.: On the extraction of atmospheric turbulence parameters from radar backscatter Doppler spectra - Theory, J. Atmos. Terr. Phys., 45, 89-102, 1983.

Hocking, W. K.: Observation and measurement of turbulence in the middle atmospfere with a VHF radar, J. Atmos. Terr. Phys., 48, 656-670, 1986.

Kelley, M. C.: The earth's ionosphere, Academic Press, San Diego, California, 1989.

Kurosaki, S., Yamanaka, M. D., Hashiguchi, H., Sato, T., and Fukao, S.: Vertical eddy diffusivity in the lower and middle atmosphere: a climatology based on the MU radar observations during 1986-1992, J. Atmos. Terr. Phys., 58, 727-734, 1996.

Nelson, S. P.: The influence of storm flow structure on hail growth, J. Atmos. Sci., 40, 1965-1983, 1983.

Nastrom, G. D.: Doppler radar spectral width broadening due to beamwidth and wind shear, Ann. Geophys., 15, 786-796, 1997, SRef-ID: 1432-0576/ag/1997-15-786. 
Nastrom, G. D., and Eaton, F. D.: Turbulence eddy dissipation rates from radar observations at 5-20 km at White Sands Missile Range, New Mexico, J. Geophys. Res., 102, 19495-19505, 1997.

Rao, T. N., Rao, D. N., and Raghavan, S.: Tropical precipitating systems observed with Indian MST radar, Radio Sci., 34, 11251139, 1999.

Rottger, J. and Larsen, M. F.: UHF/VHF radar techniques for atmospheric research and wind profiler applications, in Radar in Meteorology, edited by David Atlas, Am. Meteorol. Soc., Boston, Mass., 235-281, 1990.

Schlegel, K. and Haldoupis, C.: Observation of the modified twostream plasma instability in the midlatitude $\mathrm{E}$ region ionosphere, J. Geophys. Res., 99, 6219-6225, 1994.

Sloss, P. W. and Atlas, D.: Wind shear and reflectivity gradient effect on Doppler radar spectra, J. Atmos. Sci., 25, 1080-1089, 1968.
Spilhous, A. F.: Raindrop size, shape, and falling speed, J. Meteor., 5, 108-110, 1948

Wakasugi, K., Mizutani, A., Masaru, M., Fukao, S., and Kato, S. A direct method for deriving drop-size distribution and vertical air velocities from VHF doppler radar spectra, J. Atmos. Oceanic Technol., 3, 623-629, 1986.

Woodman, R. F. and Chu, Y. H.: Aspect Sensitivity measurements of VHF backscatter made with Chung-Li radar: Plausible mechanisms, Radio Sci., 4, 113-125, 1989.

Wang, C. Y. and Chu, Y. H.: Investigations of Blob-like Sporadic E Plasma Irregularities Using the Chung-Li VHF Radar, J. Atmos. Solar-Terr. Phys., 63, 123-13, 2001.

Ziegler, C. L., Ray, P. S., and Knight, N. C.: Hail growth in an Oklahoma multicell storm, J. Atmos. Sci., 40, 1768-1791, 1983. 